\title{
Hyponatremic Mineralocorticoid Resistance due to Urinary Tract Infection
}

National Cancer Institute

\section{Source}

National Cancer Institute. Hyponatremic Mineralocorticoid Resistance due to Urinary

Tract Infection. NCI Thesaurus. Code C126811.

A transient condition associated with urinary tract infections in infants presenting with hyponatremia and hyperkalemia, mimicking low concentrations of aldosterone. 